\title{
Simulación numérica del impacto provocado por la alteración del uso del suelo en clima termal del Valle de México
}

\author{
V. Fuentes-Gea y A. Jazcilevich-Diamant \\ División de Estudios de Posgrado, Facultad de Ingeniería, UNAM y \\ Centro de Ciencias de la Atmósfera, UNAM
}

(recibido: octubre de 2001; aceptado: diciembre de 2001)

\begin{abstract}
Resumen
Se utilizan técnicas de simulación numérica para evaluar el efecto ocasionado por la modificación del uso del suelo en el campo de temperaturas de la atmósfera del Valle de México. Los resultados sugieren que el calentamiento que ha sufrido la Ciudad de México a partir del siglo pasado se explica, no únicamente por el incremento de la zona urbana, sino también por la drástica reducción del sistema lacustre que solía existir en la región.
\end{abstract}

Descriptores: simulación, modelos, clima.

\begin{abstract}
Using numerical simulations of the thermal field variations due to land use changes in the Valley of Mexico, it is shown that the warming of the valley during the past century, could be attribute not only to the increase of the urban area, but also to the drastic decrease of the lacustrine system that used to exist.
\end{abstract}

Keywords: simulation, models, weather.

\section{Introducción}

Como resultado de la construcción de obras tendientes a minimizar el peligro de inundaciones realizadas durante los siglos XVI y XVII, se redujo el área lacustre del Valle de México de aproximadamente $1500 \mathrm{~km}^{2}$ que existían en el siglo XVI, a $1000 \mathrm{~km}^{2}$ a principios del siglo XVIII y a 120 $\mathrm{km}^{2}$ en 1929. En ese año, todavía permanecían activos algunos canales de navegación y los lagos desempeñaban un papel importante en la zona noreste del Valle de México (Escurra y Turner, 1990). La expansión demográfica y urbana que sufrió la región, principalmente durante el siglo XX, propició la desaparición de los magníficos lagos que anteriormente existieron y que dieron pie a la fundación de la Ciudad de México. Se puede afirmar que los dos factores fundamentales que han provocado cambios drásticos en los usos del suelo del Valle de México son la expansión de la zona urbana y la reducción de la antigua región lacustre.

Con base en dichos factores, este trabajo tiene como objetivo mostrar mediante la simulación numérica del campo termal de la atmósfera del Valle de México, que los cambios de los usos del suelo mencionados y principalmente los originados por la reducción de la zona lacustre, han alterado el microclima de la región. A fin de lograr lo anterior, se efectuaron una serie de experimentos numéricos que permitieron probar la bondad de los métodos 
utilizados y su capacidad de reproducir, con grados de certidumbre apropiados, el campo de temperaturas de la zona y la tendencia histórica que éste ha sufrido en los últimos 100 años. La importancia que tienen los resultados mencionados, estriba en que herramientas de simulación como las que se describen en el presente trabajo, pueden ser utilizadas para predecir el impacto de obras y procesos como el de la contaminación ambiental, así como la exploración y explotación de recursos, de modo que permitan tomar medidas para prevenir los problemas que este tipo de procesos ocasionan al ambiente.

\section{El modelo meteorológico}

El modelo utilizado en este trabajo se conoce con el nombre de MEMO (Moussiopoulos, 1987). Este modelo permite reproducir mediante simulación numérica, el campo de vientos y de temperaturas sobre orografías complejas. El modelo toma en cuenta el perfil natural del terreno usando un sistema de coordenadas que siguen las variaciones del terreno, así como características físicas del suelo que intervienen en el flujo de energía que se establece entre éste y la atmósfera.

MEMO es un modelo tridimensional no hidrostático que describe la dinámica de la capa límite atmosférica para aire no saturado. El modelo efectúa balances de masa, momentum y energía en un medio turbulento, caracterizado por flujos energéticos no estacionarios que se establecen entre la atmósfera y la superficie del suelo. Para ser más precisos, el modelo resuelve numéricamente el siguiente conjunto de ecuaciones diferenciales:

Ecuaciones de movimiento:

$$
\begin{aligned}
& \frac{\partial(\rho u)}{\partial t}+\frac{\partial(\rho u u)}{\partial x}+\frac{\partial(\rho v u)}{\partial y}+\frac{\partial(\rho w u)}{\partial z}=\frac{\partial(p)}{\partial x}+R_{u}+C_{u} \\
& \frac{\partial(\rho v)}{\partial t}+\frac{\partial(\rho u v)}{\partial x}+\frac{\partial(\rho v v)}{\partial y}+\frac{\partial(\rho w v)}{\partial z}=\frac{\partial(p)}{\partial y}+R_{v}+C_{v} \\
& \frac{\partial(\rho w)}{\partial t}+\frac{\partial(\rho u w)}{\partial x}+\frac{\partial(\rho v w)}{\partial y}+\frac{\partial(\rho w w)}{\partial z}=\frac{\partial(p)}{\partial z}+R_{w}+C_{w}-\rho g
\end{aligned}
$$

Ecuación de continuidad:

$$
\frac{\partial \rho}{\partial t}+\frac{\partial(\rho u)}{\partial x}+\frac{\partial(\rho v)}{\partial y}+\frac{\partial(\rho w)}{\partial z}=0
$$

Ecuaciones de transporte de cantidades escalares:

$$
\frac{\partial(\rho \psi)}{\partial t}+\frac{\partial(\rho u \psi)}{\partial x}+\frac{\partial(\rho v \psi)}{\partial y}+\frac{\partial(\rho w \psi)}{\partial z}=R_{\psi}+Q_{\psi}
$$

En donde $u, v$ y $w$ son las componentes de la velocidad del viento en las direcciones $x, y, z$, respectivamente, $\rho$ es la densidad del aire, $p$ la perturbación de la presión a mesoescala y $\psi$ es una cantidad escalar, tal como la humedad relativa, la temperatura potencial o la energía cinética turbulenta. Las cantidades $R_{u}, R_{v}, R_{w}$ y $R_{\psi}$ representan la difusión turbulenta de momentum y $\mathrm{C}_{u}, \mathrm{C}_{v}$ y $\mathrm{C}_{w}$ las componentes de la fuerza volumétrica de Coriolis. Los términos fuente y sumideros, representados por la cantidad $\mathrm{O}_{\psi}$ dependen de la cantidad escalar transportada. Por ejemplo, en el caso de la temperatura potencial, estos términos contienen los flujos de radiación y las emisiones antropogénicas de calor.

Las ecuaciones presentadas anteriormente, forman un sistema de ecuaciones diferenciales parciales acoplado no lineal, el cual debe resolverse mediante técnicas numéricas que permitan una solución que resulte económica. Para ello, se utilizó un esquema de discretización espacial de segundo orden, aplicado a una malla en donde las componentes de la velocidad del viento $u, v$ y $w$ se calculan en las fronteras de cada celda, mientras que las cantidades escalares, como la presión y la temperatura potencial se obtienen en sus centros. En lo que respecta a la discretización del tiempo, se empleó el esquema explícito de segundo orden de Adams-Bashforth, con excepción de los términos correspondientes a la parte no hidrostática de la perturbación de la presión y la difusión turbulenta vertical, que se manejaron mediante un método implícito. Los flujos convectivos (transporte por los vientos) se trataron por medio del esquema de Smolarkiewicz (1984), el 
cual permite reducir sustancialmente la difusión de origen numérico.

\section{Requerimientos de información del modelo}

MEMO utiliza como datos de entrada, información referente a la orografía y a los usos del suelo que caracterizan la zona de estudio, así como datos meteorológicos al inicio de la simulación y en las fronteras de la región. Con respecto a los primeros, se utilizaron datos de altura del terreno y usos del suelo en cada nodo de la malla empleada. La fiabilidad de estos datos es vital para la operación apropiada del modelo, ya que es esta información la que determina la magnitud de los flujos de energía que se establecen localmente y que originan la micro-meteorología especifica del sitio de estudio. Por otra parte, la información meteorológica que se alimentó al modelo en el inicio de la simulación, consistió en un perfil vertical de vientos, temperaturas y presiones, los cuales se interpolaron en toda la región utilizando un modelo meteorológico denominado de diagnóstico. Este es un modelo adicional que tiene como propósito interpolar el campo de vientos en toda la región, de modo que pueda constituirse en condición inicial. El proceso de interpolación que realiza dicho modelo está restringido por el principio de conservación de la masa.

En el caso específico de la aplicación de MEMO al Valle de México (Jazcilevich et al., 2000) se utilizó un sistema de mallas anidadas, en donde la malla exterior cubrió una región de $120 \times 150 \mathrm{~km}^{2}$ con una resolución de $3 \mathrm{~km}$. Como se puede apreciar en la figura 1, el dominio de cómputo envuelve una región suficientemente grande para tomar en cuenta la influencia que tiene la orografía local en generar el microclima que caracteriza a la región estudiada. Anidada a esta malla, se construyó una segunda con una cobertura de $60 \times 60 \mathrm{~km}^{2}$ y con una resolución de $1 \mathrm{~km}$. Esta segunda malla se sobrepuso prácticamente en la zona que ocupa la Ciudad de México y tuvo el propósito de lograr captar, con mayor grado de precisión, los efectos locales que genera la zona urbana a modo de que fuera posible agregarle los efectos captados por la malla exterior.
2230

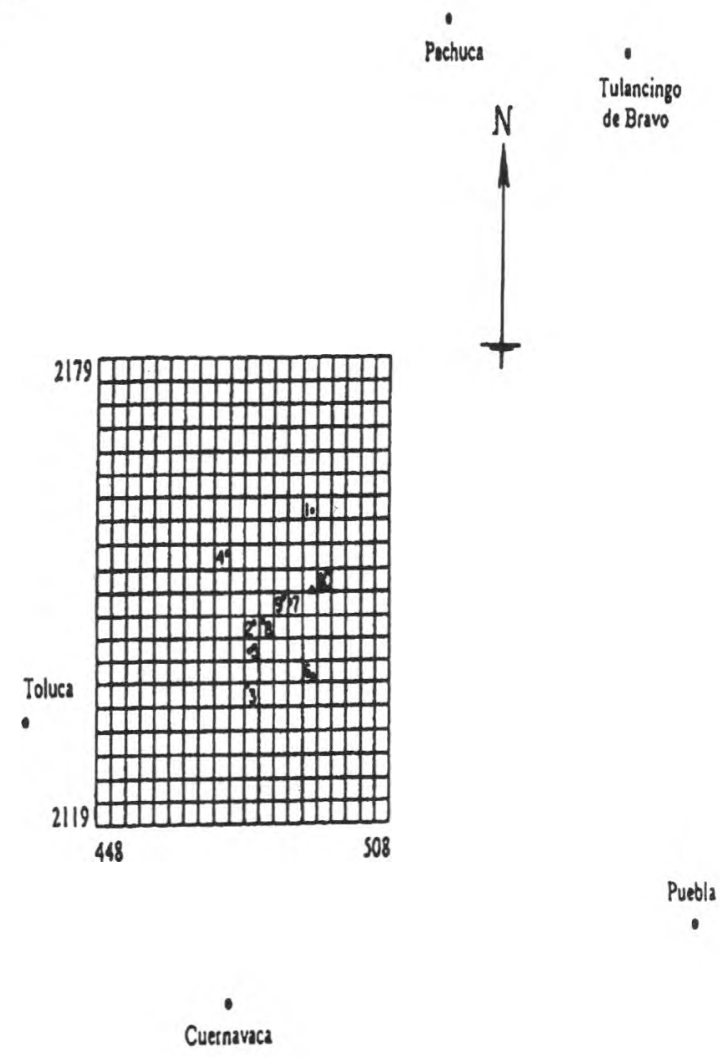

2080

430

590

Figura 1. Dominio de cómputo y ubicación de las estaciones de monitoreo en coordenadas UTM

\section{Construcción de escenarios}

Tomando en consideración que los cambios más importantes que se han llevado a cabo en la región de Valle de México en los últimos 100 años, son la reducción de la zona lacustre y el crecimiento de la mancha urbana, se armaron tres escenarios diferentes que contemplan las siguientes situaciones respecto de los usos del suelo:

1.- Usos del suelo actuales (1991)

2.- Usos del suelo de 1929

3.- Usos del suelo representando a la zona urbana de 1929 pero con los lagos actuales.

En los tres casos se empleó la misma información meteorológica, la cual correspondió al año de 1991. 
Esta información se integró con datos meteorológicos del Aeropuerto Internacional de la Ciudad de México, con los cuales se generaron los perfiles verticales de temperatura, presión y velocidades de viento necesarios, así como con datos de superficie generados en estaciones meteorológicas situadas en la ciudades de Toluca, Pachuca, Tulancingo, Puebla y Cuernavaca. Toda la información utilizada correspondió al día 22 de febrero de 1991.

Como se puede apreciar, el propósito de montar estos escenarios fue captar los cambios que ha sufrido la meteorología local, particularmente el campo termal de la zona, debido a la reducción de la región lacustre existente.

\section{Validación de modelo}

Con la finalidad de contrastar las predicciones obtenidas mediante el modelo MEMO con mediciones hechas en diferentes sitios de la zona de estudio, previo a la simulación de los escenarios ya mencionados, se seleccionaron cuidadosamente seis estaciones meteorológicas de superficie, operadas por la Red Automática de Monitoreo Atmosférica de la Ciudad de México (RAMA) y se realizó un análisis estadístico que permitió cuantificar el grado de error de las preediciones con respecto a las observaciones. Este análisis consistió en obtener en cada una de las estaciones mencionadas, el error sistemático, el error aleatorio, el coeficiente de correlación y el índice de concordancia entre mediaciones y observaciones, empleando la técnica de Willmott (1981). Los datos empleados para dicho análisis se presentan en la figura 2, en donde se muestra la evolución durante 24 horas de las temperaturas de superficie simuladas y observadas en seis estaciones situadas en la Ciudad de México. El resumen de resultados de dicho análisis se presenta en la tabla 1. En esta tabla se puede apreciar que los índices de concordancia de temperaturas superficiales fluctúan entre .86 y .98 . Es decir, se puede afirmar que en cuanto a la predicción de las temperaturas, el modelo tiende a hacer predicciones que siguen con una buena aproximación las observaciones realizadas.
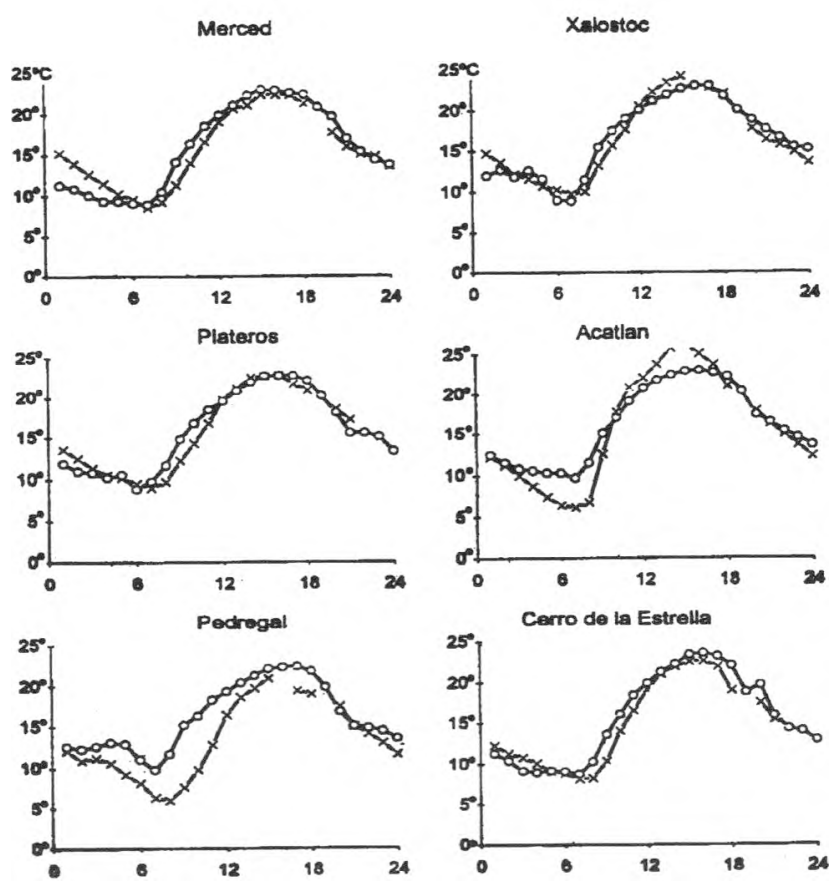

Figura 2. Temperaturas superficiales medidas (x) y simuladas (o) en diversas estaciones del Valle de México 
Tabla 1. Resumen de resultados del análisis estadístico de las temperaturas superficiales. CC coeficiente de correlación, EMC error medio cuadrático, EMCS error medio cuadrático sistemático, EMCNS error medio cuadrático no sistemático e IC índice de concordancia

\begin{tabular}{ccccccc}
\hline \hline Estación & Acatlán & Estrella & Merced & Pedregal & Plateros & Xelostoc \\
\hline CC & 0.95 & 0.93 & 0.89 & 0.79 & 0.94 & 0.91 \\
EMC & 2.60 & 1.39 & 1.52 & 3.15 & 1.31 & 1.48 \\
EMCS & 2.39 & 0.23 & 0.25 & 2.70 & 0.56 & 0.86 \\
EMCNS & 0.93 & 1.37 & 1.5 & 1.62 & 1.19 & 1.21 \\
IC & 0.94 & 0.98 & 0.97 & 0.86 & 0.98 & 0.97 \\
EMCS/EMC & 0.93 & 0.16 & 0.16 & 0.85 & 0.42 & 0.58 \\
EMCNS/EMC & 0.36 & 0.98 & 0.57 & 0.51 & 1.90 & 0.81 \\
\hline
\end{tabular}

\section{Resultados}

El resumen de los resultados de las simulaciones realizadas con los usos del suelo de 1929 (escenario 2) se presentan en la tabla 2, en donde se contrastan temperaturas máximas y mínimas, tanto medidas como simuladas, correspondientes al mes de febrero de ese año en las estaciones Tacubaya, Minería y Escandón. Como se puede apreciar en esta tabla, en Minería y Escandón se observa que las temperaturas mínimas tienden a ser mayores en la actualidad, mientras que las máximas muestran poca variación. Los resultados mencionados corresponden a temperaturas simuladas y se ven confirmados por los valores observados, los cuales presentan la misma tendencia en las tres estaciones consideradas en el estudio.

En la figura 3 y 4 se presentan isotermas promedio de temperatura potencial entre las 12:00 y las 16:00 horas, obtenidas de las simulaciones correspondientes a los tres escenarios. En la figura 4 se aprecia un patrón de distribución muy similar entre los escenarios 1 y 3 : una isoterma de $21.1 \mathrm{C}$ ocupa casi el mismo territorio en ambos casos, 10 cual muestra que si se mantiene el área urbana de 1929 intacta y se reduce el área lacustre a lo que es en este momento, se obtiene el campo de temperaturas actual. Este resultado sugiere que la reducción de la zona lacustre es suficiente para explicar los cambios de temperatura que se observan entre los escenarios 1 y 2 .

Tabla 2. Temperaturas máximas y mínimas $\left({ }^{\circ} \mathrm{C}\right)$ en las estaciones Minería, Tacubaya y Escandón

\begin{tabular}{cccccccccc}
\hline \hline Estación & \multicolumn{3}{c}{ Simulación } & \multicolumn{3}{c}{ Observación } \\
& & \multicolumn{2}{c}{1929} & & \multicolumn{2}{c}{ 1929/1930 } & & 1991 \\
\hline & Max & Min & Max & Min & Max & Min & Max & Min \\
Minería & 21.9 & 6.0 & 21.7 & 8.3 & 20.3 & 7.6 & 19.2 & 12.5 \\
Tacubaya & 21.1 & 8.6 & 21.7 & 8.6 & 24.8 & 6.4 & 24.2 & 10.1 \\
Escandón & 21.8 & 6.8 & 21.5 & 8.4 & 27.8 & 4.6 & 26.7 & 10.3 \\
\hline \hline
\end{tabular}




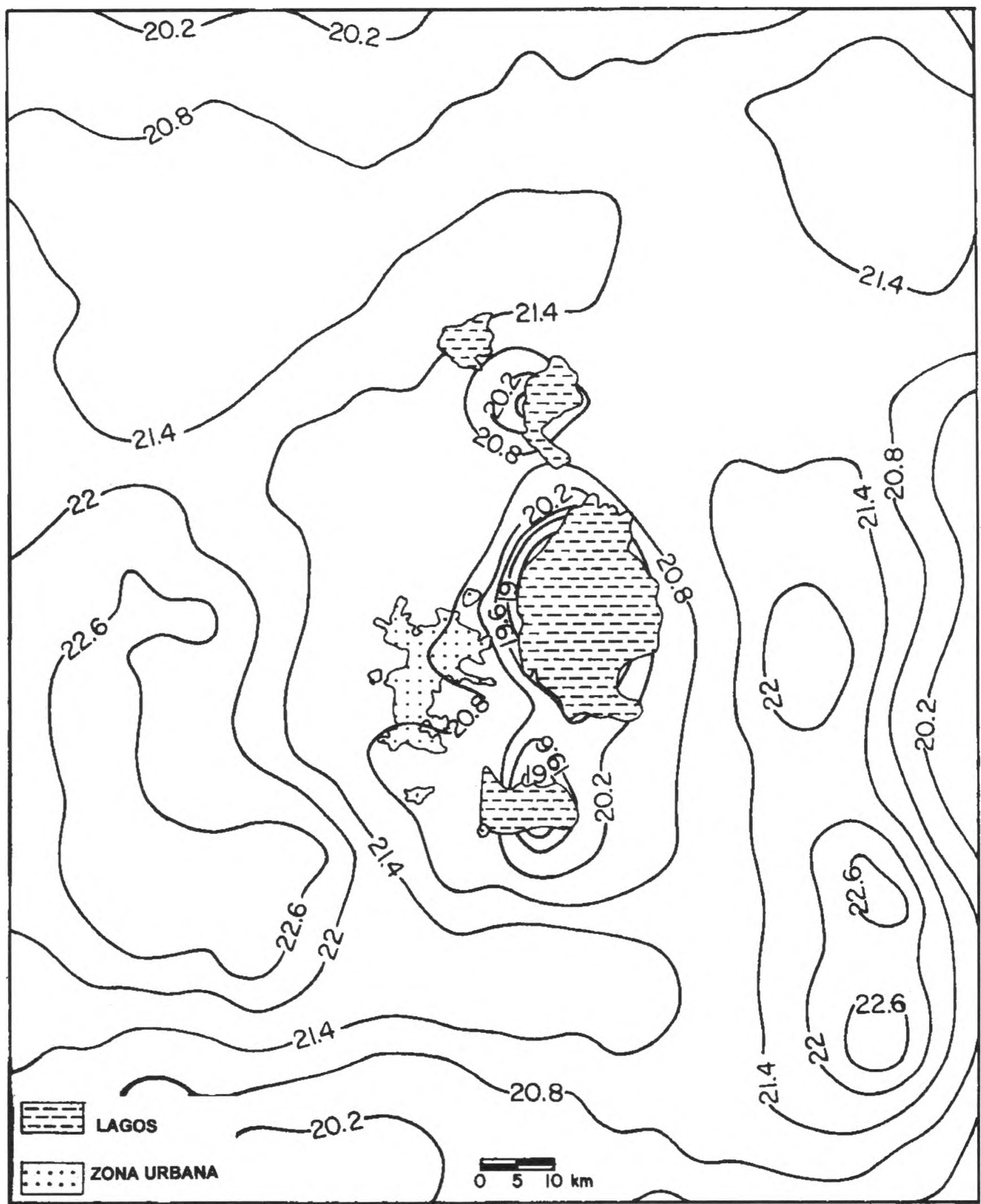

Figura 3. Isotermas de temperatura potencial entre las 12:00 y las 16:00 horas obtenidas para el escenario 2 

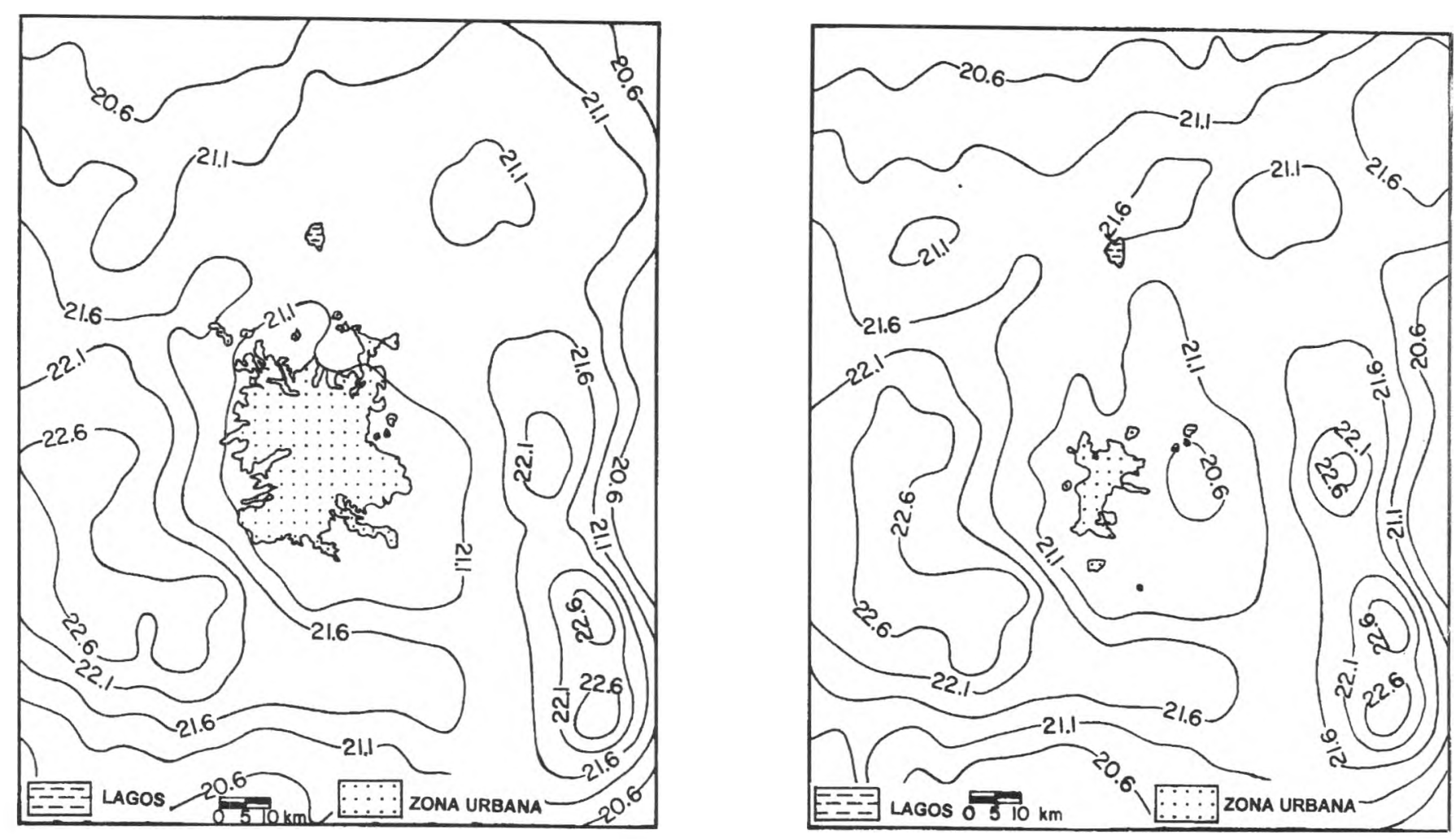

Figura 4. Isotermas de temperatura potencial entre las 12:00 y las 16:00 horas obtenidas para los escenarios 1 y 3

\section{Conclusiones}

Los dos factores fundamentales que han modificado los usos del suelo del Valle de México en los últimos 100 años son la urbanización y la desecación de los lagos existentes en la región. Con base en las simulaciones realizadas para evaluar el impacto que han tenido dichas alteraciones, se puede afirmar que éstas han tenido un efecto significativo para modificar el microclima del Valle de México. En particular, los resultados sugieren que la desecación de los lagos influyó de manera notable para propiciar el incremento de las temperaturas promedio en la zona de estudio.

La importancia que tienen las herramientas de simulación como las que se describen en el presente trabajo, es que pueden ser utilizadas para predecir el impacto de obras y procesos como el de la contaminación ambiental y la exploración y explotación de recursos y, consecuentemente, para prevenir los problemas que este tipo de procesos ocasionan al ambiente. Sin embargo, resulta imprescindible que dichas herramientas sean previamente probadas mediante técnicas estadísticas que validen los resultados obtenidos

\section{Referencias}

Escurra E. y Turner B.L. (1990). The Basin of Mexico in the Earth as Transformed by Human Action. Cambridge University Press, Cambridge UK.

lazcilevich-Diamant A., Fuentes-Gea V., láuregui O.E. y Luna E. (2000). Simulated Urban Climate Response to Historical Land Use Modifications in the Basin of Mexico. Climatic Change 4444:515-536.

Moussiopoulos N. (1987). An Efficient Scheme to Calculate Radiative Transfer in Mesoscale Models. Environmental Software, 2, 172-191.

Smolarkiewicz P.K. (1984). A Fully Multidimensional Positive Definite Advection Transport Algorithm with Small Implicit Diffusion. Journal of Computational Physics, 325-362.

Willmott C.J. (1981). On the Validation of Models.

Physical Geography, 2, 184-194, 
DOI: http://dx.doi.org/10.22201/fi.25940732e.2002.03n2.008

Semblanza de los autores

Vicente Fuentes-Gea. Físico egresado de la Facultad de Ciencias de la UNAM y maestro en ciencias en ingeniería ambiental de la Universidad de Texas en Austin. Es profesor de la División de Estudios de Posgrado de la Facultad de Ingeniería de la UNAM desde hace 25 años.

Arón Jazcilevich-Diamant. Ingeniero en computación egresado de la Facultad de Ingeniería de la UNAM y doctor en matemáticas aplicadas de la Universidad de Nueva York en Stony Brook. Actualmente es investigador en el Centro de Ciencias de la Atmósfera de la UNAM. 